Research Article

\title{
Effectiveness of local paediatric murmur clinic
}

\begin{abstract}
Background: There has been increasing work-load in Paediatric Cardiology where majority of new referrals were asymptomatic murmurs. This has inevitably caused long waiting times impacting expectations of patients and parents as well as teaching, research and service commitments at tertiary centres. To address this concern, screening clinics locally in a supported network settings were suggested.
\end{abstract}

Aim: To evaluate Local Murmur Clinic services run by a Paediatrician with Specialist Interest in Cardiology based at Royal Bolton Hospital, Bolton, United Kingdom.

Method: Retrospective review of clinic letters of patients who attended the Murmur Clinic between January and December 2013.

Findings: In total, 313 cases were reviewed. $98 \%$ of them were referred by General Practitioners and consultants. The Mean waiting time was $91 / 2$ weeks whereas the commonest waiting time (Mode) was 4 weeks. $50 \%$ of cases were aged between 6 months and 5years. Heart murmur (67\% of all cases) is the main reason of referral and $71 \%$ of these were discharged whereas $17 \%$ had abnormal Echocardiography. Overall, Cardiologist input was sought in $15 \%$ of all new cases. Murmur cases were more likely to be discharged (71\% vs $43 \%$ ) whereas other cases involved more cardiologist (27\% vs $12 \%$ ), General Paediatrics or radiology input ( $9 \%$ vs $2 \%$ ), $\mathrm{P}=0.001 .11 .5 \%$ of cases failed to attend their appointments with murmur cases being less likely to miss the appointment, $\mathrm{P}=0.001$.

Conclusion: This study demonstrated that local murmur clinic services avoid unnecessary referral of patients to tertiary centres which has dual benefits of patient convenience and reduced workload at paediatric cardiology outpatients. We recommend furtherance of supporting of the scheme and facilitation of early exposure of doctors in training to such services to enhance the likelihood of utilization of current training posts.

Keywords: paediatrician with specialist interest in cardiology, general practitioner, district general hospital, did not attend
Volume 2 Issue 2 - 2015

\author{
Esubalew Alemu,' Sameer Misra ${ }^{2}$ \\ 'Cardiothoracic Department, UHSM, UK \\ ${ }^{2}$ Paediatrics Department, Royal Bolton Hospital, UK
}

Correspondence: Esubalew Alemu, Cardiothoracic Department, UHSM, M23 9PT, United Kingdom, Tel 0161998 7070,Email esubalew@doctors.org.uk

Received: October 0I, 2014 | Published: April 24, 2015
Abbreviations: PWSIC, paediatrician with specialist interest in cardiology; GP, general practitioner; DGH, district general hospital; DNA, did not attend

\section{Introduction}

In the UK, there are 15 paediatric Cardiology centres supporting approximately 200 District General Hospitals (DGHs). ${ }^{1}$ There is a general impression that the demands at paediatric cardiology outpatient clinic have significantly increased over the previous years due to reasons such as increased pick up rate of asymptomatic cardiac murmurs by medical practitioners and parental demand for reassurance. ${ }^{2,3}$ Consequently, the increased workload at tertiary centres has been thought to have an impact on the patient care, teaching and research as senior doctors become unable to undertake these commitments. ${ }^{4}$

Therefore, it has been a longstanding view amongst paediatric medical community in the United Kingdom to establish local murmur clinics which serve as 'screening clinics' providing echocardiography service led by a paediatrician with specialist interest in cardiology (PWSIC). The service is thought to enable discharging patients referred with murmur but found to have normal cardiac structure prior to referral to a cardiologist thereby freeing up clinic slots at tertiary centres. For example, Pushparajah et al., ${ }^{2}$ demonstrated that such clinic reduced unnecessary referral to specialist centres by $21 \%$. Additionally, local clinics were deemed to be more convenient for the patients and their families avoiding long distance journeys to tertiary centres and reducing clinic waiting times.
This article reports the findings of evaluation of local murmur clinic services set up in line with the Pushparajah K et al., ${ }^{2}$ working model of care.

\section{Aim}

To evaluate Bolton Local Murmur Clinic services based at Royal Bolton Hospital, United Kingdom during the period of January 2013 to December 2013 so as to assess the effectiveness of achieving its primary goal of supporting local community and tertiary centres.

\section{Setting}

Bolton Local Murmur Clinic at the Royal Bolton Hospital is run by a paediatrician with specialist interest in cardiology (PWSIC). There are 56 clinics per year: 36 on Monday and 20 on Tuesday where local referrals from various health care professionals are accepted and on average, 8-10 patients per clinic attend. Echocardiographic scanning is conducted and patients are triaged according to the findings.

\section{Method}

A retrospective review of clinic letters of patients who attended the murmur clinic during the lyear period was performed by the author. The letters were accessed via the IPM software where the electronic copies of the letters were stored. Data was collected using a proforma designed on Excel Spreadsheet and statistical analysis of the data matrix was done with the SPSS software and P value of 0.05 was considered to be statistically significant. The values are reported 
in percentages and rounded off to the nearest whole number where appropriate.

\section{Findings}

In total, 324 appointments were booked during this period and clinic letters of 11 cases were not accessible via the software. The remaining 313 cases were reviewed. 263 (84\%) of these appointments were new and $50(16 \%)$ were follow up. $37(11.5 \%)$ failed to attend their appointments (Table 1).

Table I This table shows the number (percentage) of the new cases, follow up cases and those who did not attend

\begin{tabular}{llll}
\hline Total & 324 & Unaccessible & I I \\
\hline Reviewed Total & 313 & \\
New Cases & $263(84 \%)$ & \\
Follow Up Cases & $50(16 \%)$ & \\
Did Not Attend (DNA) & $37(1 \mathrm{I} .5 \%)$ & \\
\hline
\end{tabular}

\section{Waiting time}

The mean waiting time for new appointments was 9.56weeks with the median and mode of 8 and 4weeks respectively. The waiting time ranges from being seen on the same day of referral to 52 weeks.

\section{Age}

The age range of new cases is between 3 days and 16years of age. The distribution of the age group of the new cases is demonstrated in Figure 1 .

\section{Sources of referral}

$57 \%(\mathrm{~N}=150)$ of new cases were referred by consultants which include paediatricians, neonatologists and Accident and Emergency consultants. 41\% ( $\mathrm{N}=108)$ were referred by General Practitioners (GPs) whereas the remaining $2 \%(\mathrm{~N}=5)$ were referred by multidisciplinary team such as advanced practitioners and technicians.

\section{Referral reason}

The commonest reason of referral the new cases was cardiac murmur $67 \%(\mathrm{~N}=176)$ and the remaining $33 \%(\mathrm{~N}=87)$ include chest pain, palpitations, fainting, family history of cardiac diseases, abnormal ECGs etc Figure 2.

\section{Echocardiography findings}

All patients had echocardiography and $71 \%(\mathrm{~N}=186)$ of new cases had normal echocardiography results whereas $14 \%(\mathrm{~N}=36)$ had abnormal findings. The remaining $16 \%(\mathrm{~N}=41)$ include those who did not attend or cooperate with the scanning requiring a follow up arrangement. When comparing those with murmur to those without murmur, $73 \%(\mathrm{~N}=129)$ and $77 \%(\mathrm{~N}=55)$ had normal echocardiography respectively whereas $17 \%(\mathrm{~N}=30)$ and $9 \%(\mathrm{~N}=6)$ had abnormal echocardiography respectively. Hence, greater proportion of those with murmur had abnormal echocardiography, nonetheless, there was no statistical significance $(\mathrm{P}=0.166)$.

\section{Outcome}

$60 \%(\mathrm{~N}=158)$ of the new cases were discharged from the local murmur clinic whereas a cardiologist input was sought in $15 \%(\mathrm{~N}=40)$ and $9.9 \%(\mathrm{~N}=26)$ had murmur clinic follow up (Figure 3$)$.

Among those referred with murmur, $71 \% \quad(\mathrm{~N}=125)$ were discharged, $12 \%(\mathrm{~N}=21)$ had a cardiologist input, $10 \%$ had local murmur clinic follow up, $6 \%(\mathrm{~N}=10)$ did not attend and $1.7 \%(\mathrm{~N}=3)$ had other outcome such as repeat echocardiography for being upset during examination. In contrast, in those referred with other causes, $43 \%(\mathrm{~N}=30)$ were discharged, $27 \%(\mathrm{~N}=19)$ had a cardiologist input, $10 \%(\mathrm{~N}=8)$ had local murmur clinic follow up, $10 \%(\mathrm{~N}=7)$ did not attend and $9 \%(\mathrm{~N}=6)$ had other outcomes which include general paediatric follow up or radiology team input. This shows that those referred with murmur were more likely to be discharged and less likely to involve a cardiologist or fail to attend, $\mathrm{P}=0.001$ (Figure 4).

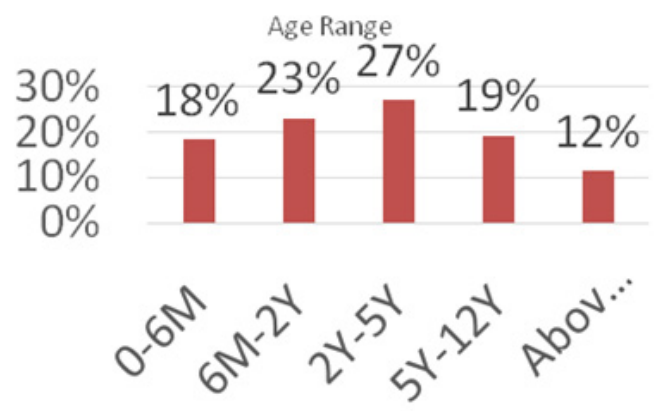

Figure I The percentage of age groups seen in the clinic.

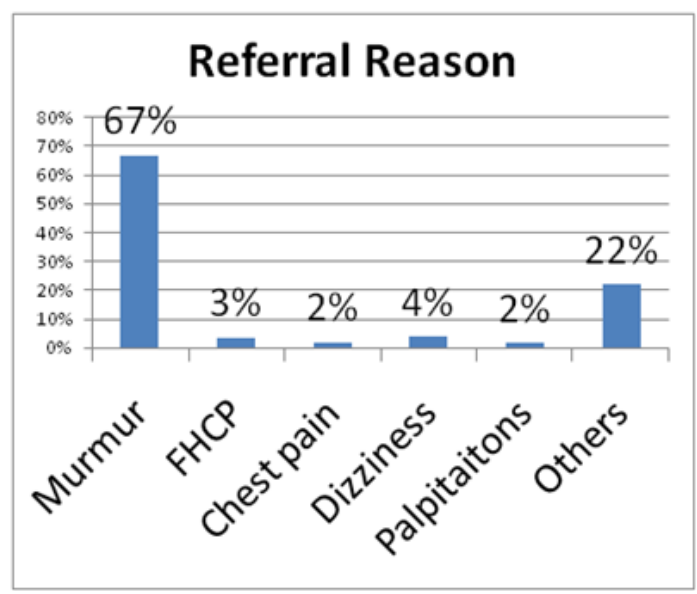

Figure 2 The percentage of causes of referral. Others refers to a long list including screening for conditions with chromosomal abnormalities, Kawasaki Disease, Marfan's syndrome etc.

FHCP - Family History of Cardiac Pathology.

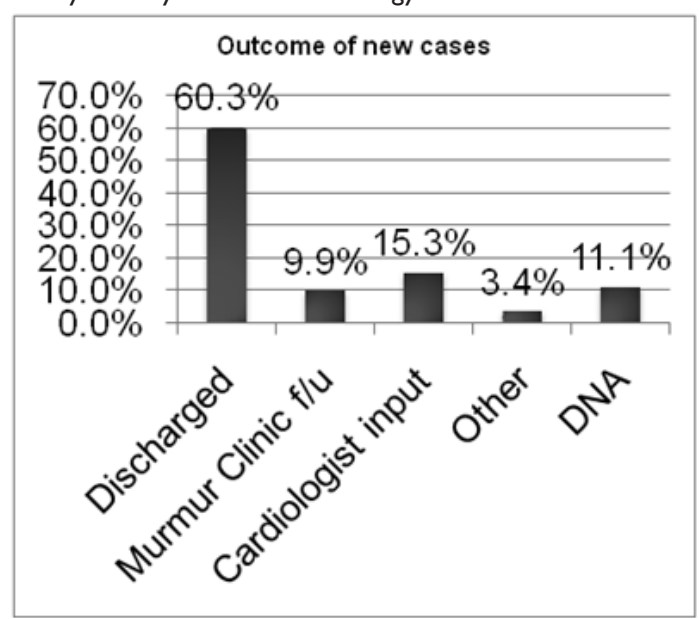

Figure 3 Demonstrates the percentage of outcome of the new cases seen at the clinic. Other includes general paediatric follow up and radiologist input. Where, Murmur Clinic f/u: Murmur Clinic Follow Up; DNA: Did Not Attend. 


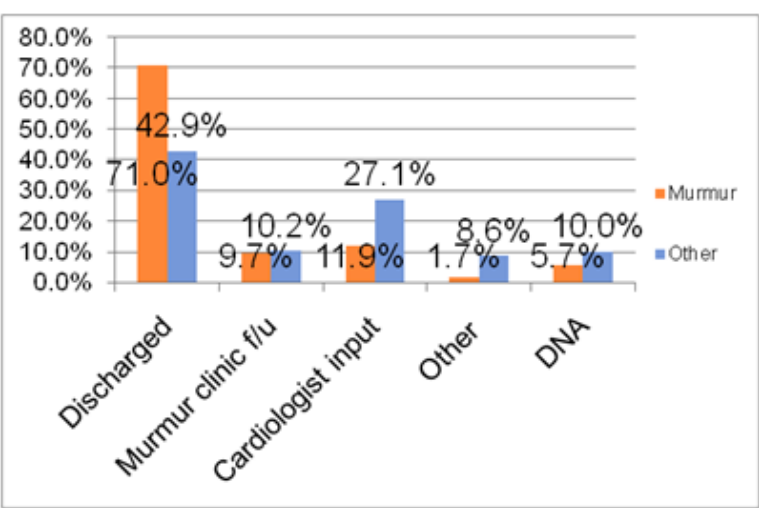

Figure 4 Displays the percentages of the outcome of those referred with murmur and without murmur.

Murmur clinic f/u: Murmur Clinic Follow Up; DNA: Did Not Attend.

When analysing those who did not attend their appointments $(11.5 \%, \mathrm{~N}=36), 81 \%(\mathrm{~N}=29)$ of these were new cases whilst $19 \%$ $(\mathrm{N}=7)$ were follow up cases. $11.7 \%(\mathrm{n}=15)$ of cases referred by Paediatricians (total $=128)$ did not attend whereas $10.2 \% \quad(n=11)$ of those referred by GPs (total=108) did not attend. The difference between the two groups was not statistically significant $(\mathrm{P}=0.475)$.

\section{Discussion}

The majority of cases were referred with murmur and $17 \%$, that is 1 in 6 of these had abnormal echocardiography which includes findings such as ventricular septal defect, atrial septal defect, coarctation of aorta etc. That means when identifying cardiac murmur in a child during physical examination, there is a 1 in 6 chance that could be pathological. Another study documented that $60 \%$ of neonates seen at cardiac clinic with murmur had cardiac defect indicating that murmur can be a sign of underlying cardiac pathology. ${ }^{5}$ Thus, it appears appropriate to refer these cases for further investigation.

Notwithstanding this, $60 \%$ of all the new appointments were discharged and cardiologist input was sought only in $15 \%$ of the new cases. Besides, $71 \%$ of the murmur patients were discharged and lesser proportion of these group (12\%) needed cardiologist input. This indicates that this clinic is serving its primary purpose of avoiding tertiary referrals whilst being situated in the local vicinity. On the other hand, only $43 \%$ of those without murmur were discharged despite $77 \%$ of them having normal echocardiography findings. This is likely due to a greater proportion of those without murmur in comparison with those with murmur tend to involve cardiologist input, need General Paediatric follow up or radiology team meetings $(\mathrm{P}=0.001)$

$11.5 \%$ did not attend their appointments with no difference in DNA rate in relation to the referral source. This raises the importance of reiterating attendance by all teams when referring patients especially those without murmur as this group is more likely to fail to attend the appointments (10\% vs 6\%) yet require further follow up. Attendance of those with murmur seems to be better as they might be more worried than those without murmur.

The mean waiting time was nine and half weeks signifying the high demands on the service. And also, broad age groups of paediatric patients attended the clinic which poses some challenges from equipment standpoint as several echocardiography probes are needed. A recent survey revealed that only $35 \%$ of the DGHs in the UK were noted to have a PWSIC which hints some variability in services across the UK. ${ }^{6}$ In order for the service to gain its full momentum, further efforts to implement the current working model and promote awareness of its advantages need to be orchestrated. For instance, releasing a PWSIC to run these clinics or invest in recruiting new personnel could reduce waiting times. Moreover, the collaboration between the PWSICs and tertiary centres in terms of audits, support network and continued professional development is vital to sustain the service. For junior doctors, facilitation of placements and early exposure in these clinics would enhance the utilization of designated training posts available nationally. ${ }^{7}$

Finally, in this work, inaccessibility of 11 clinic letters via the IPM software and the availability of limited information on clinic letters as compared to the full medical notes of the patients impose some limitation. However, large data was collected and the author performed data gathering and interpretation under continued supervision of the same consultant which reduces variability. The findings of this work strengthen the recent notion of the scientific community that local murmur clinics are beneficial. This document posits the outcome based on the data obtained from one local murmur clinic. In the future, a multicentre scrutiny of the measures including patients and families satisfaction in relation to murmur clinics could produce further robust evidence.

\section{Conclusion}

A local murmur clinic run by an appropriately trained and supported paediatrician with specialist interest in cardiology efficiently avoids unnecessary referral of innocent murmurs to tertiary centres whilst creating a local setting for those who need further follow up. Hence, the service warrants widening of support with emphasis on logistics and familiarization of junior doctors with such clinics at the earliest opportunity.

\section{Acknowledgments}

None.

\section{Conflicts of interest}

Author declares there are no conflicts of interest.

\section{Funding}

None.

\section{References}

1. Dowie R, Mistry H, Rigby M, et al. A paediatric telecardiology service for district hospitals in south-east England: an observational study. Arch Dis Child. 2009;94(4):273-277.

2. Pushparajah K, Garvie D, Hickey A, et al. Managed Care Network for the assessment of cardiac problems in children in a district general hospital: a working model. Arch Dis Child. 2006;91(11):892-895.

3. Tybulewicz AT, Rigby ML, Redington AN. Open-access paediatric echocardiography: changing role and referral patterns to a consultant-led service in a tertiary referral centre. Heart. 1996;75(6):632-634.

4. Wagstaff M, Rigby M, Redington A. Increasing workload and changing referral patterns in paediatric cardiology outreach clinics: implications for consultant staffing. Heart. 1998;79(3):223-224.

5. Patton C, Hey E. How effectively can clinical examination pick up congenital heart disease at birth? Arch Dis Child Fetal Neonatal Ed . 2006;91(4):F263-F267. 
6. Karuppaswamy V, Kelsall W. Review of paediatric cardiology services in district general hospitals in the United Kingdom. Arch Dis Child. 2009;94(4):327.

7. Gibbs JL. Paediatricians subspecialising in cardiology: clinical governance, maintenance of expertise, and training. Arch Dis Child. 2006;91(11):878-879. 